\title{
ON THE CONVEXITY OF MEAN VALUE FUNCTIONS
}

\section{HAROLD SHNIAD}

1. Introduction. Let $(a)$ denote a set $a_{1}, a_{2}, \cdots, a_{n}$ of $n$ distinct positive numbers, $n \geqq 2$, with the subscripts $\nu$ labeled so that $a_{\nu}<a_{\nu+1}$ for $\nu=1, \cdots, n-1$. Let $(\xi)$ denote a set of positive numbers $\xi_{1}, \xi_{2}, \cdots, \xi_{n}$ with $\sum_{\nu=1}^{n} \xi_{\nu}=1$. The mean value function $M_{t}(a, \xi)$ $=\left(\sum_{\nu=1}^{n} \xi_{\nu} a_{\nu}^{t}\right)^{1 / t}, \quad t \neq 0, \quad \pm \infty ; \quad M_{0}(a, \quad \xi)=\coprod_{\nu=1}^{n} a_{\nu}^{\xi_{\nu}} ; \quad M_{-\infty}(a, \quad \xi)$ $=\min _{\nu=1,2, \cdots, n} a_{\nu}$ and $M_{+\infty}(a, \xi)=\max _{\nu=1,2, \cdots, n} a_{\nu}$; is a continuous and strictly increasing function of $t$ for $-\infty \leqq t \leqq+\infty .{ }^{1}$ For given fixed sets $(a)$ and $(\xi)$, let $M(t)$ denote $M_{t}(a, \xi)$ and $\Lambda(t)$ denote $\log M_{t}(a, \xi)$. Each of the functions $M(t)$ and $\Lambda(t)$ has horizontal asymptotes and consequently at least one point of inflection. We shall show that these functions may have more than one inflection point, but shall show that $\Lambda(t)$ is a convex function of $t$ in a neighborhood of $-\infty$, and a concave function of $t$ in a neighborhood of $+\infty$. A sufficient condition will be obtained for $\Lambda(t)$ to be convex for all negative $t$, and one for $\Lambda(t)$ to be concave for all positive $t$. Finally, the applicability of the methods used to more general weighted sums will be considered briefly.

2. Notations and fundamental formulae. Let

$$
\begin{aligned}
f(t) & =\log \left(\sum_{\nu=1}^{n} \xi_{\nu} a_{\nu}^{t}\right) \\
\eta_{\nu}(t) & =\frac{\xi_{\nu} a_{\nu}^{t}}{\sum_{\nu=1}^{n} \xi_{\nu} a_{\nu}^{t}}, \lambda_{\nu}=\log a_{\nu} \quad(\nu=1,2, \cdots, n), \\
S_{k} & =\sum_{\nu=1}^{n} \eta_{\nu}\left(\lambda_{\nu}\right)^{k} .
\end{aligned}
$$

Then

$$
\frac{d \eta_{\nu}}{d t}=\eta_{\nu} \lambda_{\nu}-\eta_{\nu} S_{1}, \frac{d S_{k}}{d t}=S_{k+1}-S_{1} S_{k}
$$

Presented to the Society, December 31, 1947; received by the editors July 5, 1947, and, in revised form, August 25, 1947.

1. See Hardy, Littlewood, and Polya, Inequalities, Cambridge University Press, 1934, chap. 2, for the basic properties of the mean value function. 


$$
\begin{aligned}
f^{\prime} & =S_{1}, \\
f^{\prime \prime} & =S_{2}-S_{1}^{2}, \\
f^{\prime \prime \prime} & =S_{3}-3 S_{1} S_{2}+2 S_{1}^{3}, \\
f^{\mathrm{IV}} & =S_{4}-4 S_{1} S_{3}-3 S_{2}^{2}+12 S_{1}^{2} S_{2}-6 S_{1}^{4}, \\
f^{\mathrm{IV}} & =\left(S_{4}-3 S_{2}^{2}+2 S_{1}^{4}\right)-4 S_{1} f^{\prime \prime \prime} .
\end{aligned}
$$

Since $\Lambda(t)=\log M(t)=f / t$, we have

$$
\Lambda^{(k)}=(1 / t)\left(f^{(k)}-k \Lambda^{(k-1)}\right) ;
$$

letting $k=3$ in (6) and integrating, we obtain the result:

$$
\left(t_{2}^{3}\right) \Lambda^{\prime \prime}\left(t_{2}\right)=\left(t_{1}^{3}\right) \Lambda^{\prime \prime}\left(t_{1}\right)+\int_{t_{1}}^{t_{2}} t^{2} f^{\prime \prime \prime} d t
$$

or

$$
t^{3} \Lambda^{\prime \prime}(t)=\int_{0}^{t} t^{2} f^{\prime \prime \prime} d t \text { and } \Lambda^{\prime \prime}(0)=(1 / 3) f^{\prime \prime \prime}(0) .
$$

3. The function $f^{\prime \prime \prime}(t)$. The idea behind our analysis of the convexity or concavity of $\Lambda(t)$ in certain ranges of $t$ is to deduce results from corresponding properties of the relatively simpler function $f^{\prime \prime \prime}(t)$. By comparing formula (4) with the following expression:

$$
\Lambda^{\prime \prime}(t)=(1 / t)\left(S_{2}-S_{1}^{2}\right)-\left(2 / t^{2}\right)\left(S_{1}\right)+\left(2 / t^{3}\right) f,
$$

it may be seen that a direct study of $\Lambda^{\prime \prime}(t)$ is considerably more complicated than that of $f^{\prime \prime \prime}(t)$. Formulae (7) and (8) will be used to pass from conclusions regarding the sign of $f^{\prime \prime \prime}(t)$ in an interval to similar conclusions regarding the sign of $\Lambda^{\prime \prime}(t)$.

Our analysis of $f^{\prime \prime \prime}(t)$ will be that of a function:

$$
F(t)=\sum_{\nu=1}^{n} \eta_{\nu}\left(\lambda_{\nu}\right)^{3}-3\left(\sum_{\nu=1}^{n} \eta_{\nu} \lambda_{\nu}\right)\left[\sum_{\nu=1}^{n} \eta_{\nu}\left(\lambda_{\nu}\right)^{2}\right]+2\left(\sum_{\nu=1}^{n} \eta_{\nu} \lambda_{\nu}\right)^{3},
$$

where the expressions $\eta_{\nu}, \lambda_{\nu}(\nu=1,2, \cdots, n ; n \geqq 2)$ are subject to the conditions:

(a) $\lambda_{\nu}$ is any fixed finite valued real number with $\lambda_{\nu}<\lambda_{\nu+1}$;

(b) $\eta_{\nu}=\eta_{\nu}(t)$ with $\eta_{\nu}>0, \sum_{\nu=1}^{n} \eta_{\nu}(t)=1$, and $\eta_{\nu}(t)$ is a continuous function of $t$ defined for all real $t$;

(c) $\eta_{1}$ is a strictly decreasing function of $t$ and $\lim _{t \rightarrow-\infty} \eta_{1}=+1$, $\lim _{t \rightarrow+\infty} \eta_{1}=0$;

(d) $\eta_{n}$ is a strictly increasing function of $t$ and $\lim t \rightarrow-\infty \eta_{n}=0$, $\lim _{t \rightarrow+\infty} \eta_{n}=+1$. 
That $f^{\prime \prime \prime}(t)$ is a function $F(t)$ follows easily from formula (4) and the definitions (2) and (3).

LEMma. The function $F(t)$ has the property that if each $\lambda_{\nu}$ is replaced by $\lambda_{\nu}+c$ ( $c$ independent of $\nu$ ), then $F(t)$ remains invariant.

PRoof. Let $S_{k}(t, c)=\sum_{\nu=1}^{n} \eta_{\nu}\left(\lambda_{\nu}+c\right)^{k}$. Then

$$
\frac{\partial S_{k}(t, c)}{\partial c}=k S_{k-1}(t, c), \quad \frac{\partial S_{1}(t, c)}{\partial c}=1,
$$

and

$$
\frac{\partial}{\partial c}\left\{S_{3}(t, c)-3 S_{1}(t, c) S_{2}(t, c)+2\left[S_{1}(t, c)\right]^{3}\right\}=0 .
$$

Theorem 1. (a) If $\eta_{1}\left(t_{1}\right)=1 / 2$, then $t<t_{1}$ implies that $F(t)>0$.

(b) If $\eta_{n}\left(t_{2}\right)=1 / 2$, then $t>t_{2}$ implies that $F(t)<0$.

(c) We have $F\left(t_{1}\right)=0$ if and only if $n=2$; that is, if and only if $t_{1}=t_{2}$.

Proof. Case $1, n=2$. We replace $\lambda_{\nu}$ by $\lambda_{\nu}-\lambda_{2}$ and obtain $F(t)$ $=\left(\eta_{1}-3 \eta_{1}^{2}+2 \eta_{1}^{3}\right)\left(\lambda_{1}-\lambda_{2}\right)^{3}=\left(1-2 \eta_{1}\right) N(t)$, where $N(t)<0$. Hence $F(t)>0$ for $t<t_{1}$, since $\eta_{1}(t)$ is a strictly decreasing function of $t$.

Case $2, n>2$. Assume the theorem valid for all $n$ satisfying $2 \leqq n \leqq k$ and consider $n=k+1$. Suppose that $\eta_{1}(t)>1 / 2$, and let $d_{\nu}=\lambda_{\nu}-\lambda_{2}$. Then

$$
\begin{aligned}
F(t)= & \left(\eta_{1} d_{1}^{3}+\sum_{\nu=3}^{k+1} \eta_{\nu} d_{\nu}^{3}\right)-3\left(\eta_{1} d_{1}+\sum_{\nu=3}^{k+1} \eta_{\nu} d_{\nu}\right)\left(\eta_{1} d_{1}^{2}+\sum_{\nu=3}^{k+1} \eta_{\nu} d_{\nu}^{2}\right) \\
& +2\left(\eta_{1} d_{1}+\sum_{\nu=3}^{k+1} \eta_{\nu} d_{\nu}\right)^{3} \\
= & P_{1}+P_{2}+P_{3}+P_{4},
\end{aligned}
$$

where

$$
\begin{aligned}
& P_{1}=\left(\eta_{1}-3 \eta_{1}^{2}+2 \eta_{1}^{3}\right) d_{1}^{3}, \\
& P_{2}=\sum_{\nu=3}^{k+1} \eta_{\nu} d_{\nu}^{3}-3\left(\sum_{\nu=3}^{k+1} \eta_{\nu} d_{\nu}\right)\left(\sum_{\nu=3}^{k+1} \eta_{\nu} d_{\nu}^{2}\right)+2\left(\sum_{\nu=3}^{k+1} \eta_{\nu} d_{\nu}\right)^{3}, \\
& P_{3}=3 \eta_{1} d_{1}^{2}\left(2 \eta_{1}-1\right) \sum_{\nu=3}^{k+1} \eta_{\nu} d_{\nu}, \\
& P_{4}=3 \eta_{1} d_{1}\left[2\left(\sum_{\nu=3}^{k+1} \eta_{\nu} d_{\nu}\right)^{2}-\sum_{\nu=3}^{k+1} \eta_{\nu} d_{\nu}^{2}\right] .
\end{aligned}
$$


We have $P_{1}>0$ for $\eta_{1}>1 / 2$, since $d_{1}<0$.

If we regard the numbers $0, d_{3}, \cdots, d_{k+1}$ as $\lambda$ 's and the numbers $\left(\eta_{1}+\eta_{2}\right), \eta_{3}, \cdots, \eta_{k+1}$ as weights, then the expression $P_{2}$ is a function $F(t)$ with $n=k$. Since $\left(\eta_{1}+\eta_{2}\right)>1 / 2$ whenever $\eta_{1}>1 / 2$, the induction hypothesis implies that $P_{2}>0$.

We have $P_{3}>0$ since $d_{\nu}>0$ for $\nu=3, \cdots, k+1$.

Cauchy's inequality gives:

$$
\left(\sum_{\nu=3}^{k+1} \eta_{\nu} d_{\nu}\right)^{2} \leqq\left(\sum_{\nu=3}^{k+1} \eta_{\nu} d_{\nu}^{2}\right)\left(\sum_{\nu=3}^{k+1} \eta_{\nu}\right)<\frac{1}{2}\left(\sum_{\nu=3}^{k+1} \eta_{\nu} d_{\nu}^{2}\right) .
$$

This inequality together with $d_{1}<0$ gives $P_{4}>0$. This concludes the proof of part (a).

If $\eta_{1}=1 / 2$ and $n>2$, then $P_{1}=P_{3}=0, P_{2}>0$ and $P_{4}>0$. Hence for $n>2$, we have $F\left(t_{1}\right)>0$. The rest of the theorem may be verified by using a similar procedure (for example, replace $\lambda_{\nu}$ by $\lambda_{\nu}-\lambda_{k-1}$, and so on).

4. The behavior of $\Lambda^{\prime \prime}(t)$. We shall establish the following result:

THEOREM 2. There exists a $t_{1}$ such that $t<t_{1}$ implies that $\Lambda^{\prime \prime}(t)>0$.

Proof. By Theorem 1 and the fact that $\eta_{1}$ decreases continuously from plus 1 to 0 , there is a value $p$ such that $f^{\prime \prime \prime}(p)=0$ and such that $t<p$ implies $f^{\prime \prime \prime}(t)>0$.

(a) If $p>0$, then $\Lambda^{\prime \prime}(t)>0$ for all $t$ on the range $-\infty<t \leqq p$. Since $\Lambda^{\prime \prime}(0)=(1 / 3) f^{\prime \prime \prime}(0)$, we have $\Lambda^{\prime \prime}(0)>0$. That $\Lambda^{\prime \prime}(t)>0$ for all nonzero $t$ on the given range follows from formula (8).

(b) If $p=0$, then $\Lambda^{\prime \prime}(0)>0$ for all negative $t$, and $\Lambda^{\prime \prime}(0)=0$.

(c) If $p<0$ and $\Lambda^{\prime \prime}(\bar{t}) \leqq 0$ for some $\bar{t}<p$, then from formula (7) we have $\Lambda^{\prime \prime}(t)<0$ for all $t$ satisfying $\bar{t}<t \leqq p$. But $\Lambda(t)$ is an increasing and bounded function of $t$. Hence for some $t_{1} \leqq p, \Lambda^{\prime \prime}\left(t_{1}\right)>0$ and for such a $t_{1}, t<t_{1}$ will imply that $\Lambda^{\prime \prime}(t)>0$.

CoRollary 1. There exists a $t_{1}$ such that $M(t)$ is convex for $t \leqq t_{1}$.

Corollary 2. If $\xi_{1} \geqq 1 / 2$, then both $\Lambda(t)$ and $M(t)$ are convex for all negative $t$.

Proof. Since $\eta_{1}(0)=\xi_{1}$, the inequality $\xi_{1} \geqq 1 / 2$ implies that $f^{\prime \prime \prime}(t)>0$ for all negative $t$. The conclusion for $\Lambda(t)$ follows from parts (a) and (b) of the proof of Theorem 2. Since the convexity of the logarithm of a function always implies the convexity of the function, the result for $M(t)$ follows.

The same methods may be used to prove: 
THEOREM $2^{\prime}$. There exists a $t_{2}$ such that $t>t_{2}$ implies that $\Lambda^{\prime \prime}(t)<0$.

Corollary $2^{\prime}$. If $\xi_{n} \geqq 1 / 2$, then $\Lambda(t)$ is concave for all positive $t$.

THEOREM 3. If $f^{\prime \prime \prime}(t)$ has exactly one zero, then so does $\Lambda^{\prime \prime}(t)$.

Proof. Let $f^{\prime \prime \prime}(\bar{t})=0$. Then we have $f^{\prime \prime \prime}(t)>0$ for $t<\bar{t}$ and $f^{\prime \prime \prime}(t)<0$ for $t>\bar{t}$. If $\bar{t}>0$, the proofs of Theorems 2 and $2^{\prime}$ show that $\Lambda^{\prime \prime}(t)>0$ on the range $-\infty<t \leqq \vec{t}$ and that $\Lambda^{\prime \prime}(t)$ has one zero on the range $\bar{t}<t<+\infty$. The other cases $\bar{t}=0$ and $\bar{t}<0$ are similar.

5. Convex-concave functions. A function $g(t)$ defined on the range $-\infty<t<+\infty$ is convex-concave providing there exists a point $\bar{t}$ such that $g(t)$ is convex for $t \leqq \bar{t}$ and concave for $\bar{t} \leqq t$.

The question may be raised whether either of the functions $\Lambda(t)$ or $M(t)$ is necessarily convex-concave. By Theorems 1 and 3 the answer is affirmative for $\Lambda(t)$ in the case $n=2$. We now develop an example to show that this conclusion regarding either $\Lambda(t)$ or $M(t)$ is not true for $n>2$.

Consider the case $a_{\nu}=e^{\nu}(\nu=1,2,3), \xi_{1}=\xi_{3}<1 / 6, \lambda_{\nu}=\nu$. If $\lambda_{\nu}$ is replaced by $\lambda_{\nu}+c, f^{\prime}(t)$ is increased by $c$ and every higher order derivative of $f(t)$ remains invariant. Therefore, to calculate both $f^{\prime \prime \prime}(0)$ and $f^{\mathrm{IV}}(0)$, the set $(1,2,3)$ may be replaced by the set $(-1,0,+1)$. We have that $f^{\prime \prime \prime}(0)$ is equal to $-\xi_{1}+\xi_{3}=0$ and, by formula (5), that $f^{\mathrm{IV}}(0)$ is equal to $\left(\xi_{1}+\xi_{3}\right)-3\left(\xi_{1}+\xi_{3}\right)^{2}>0$. Therefore in a neighborhood of zero, $f^{\prime \prime \prime}(t)$ has the same sign as $t$. From formula (8), it follows that $\Lambda^{\prime \prime}(t)$ agrees with $f^{\prime \prime \prime}(t)$ in sign throughout this neighborhood and consequently that $\Lambda^{\prime \prime}(t)$ has at least three zeros. In particular for the mean value function $M(t)=\left(.1 e^{t}+.8 e^{2 t}+.1 e^{3 t}\right)^{1 / t}$, the following table shows that there are values $t_{1}$ and $t_{2}$ such that $t_{1}<t_{2}$ and $M^{\prime \prime}\left(t_{1}\right)<0<M^{\prime \prime}\left(t_{2}\right)$ or that $M(t)$ is not convex-concave.

Table of $M$ and its derivatives for $M(t)=\left(.1 e^{t}+.8 e^{2 t}+.1 e^{3 t}\right)^{1 / t}$

\begin{tabular}{l|cccc}
\hline \multicolumn{1}{c|}{$t$} & -1.2 & -0.8 & -0.4 & 0.0 \\
\cline { 2 - 5 }$M(t)$ & 6.519 & 6.810 & 7.098 & 7.389 \\
$M^{\prime}(t)$ & 0.7311 & 0.7215 & 0.7209 & 0.7389 \\
$M^{\prime \prime}(t)$ & -0.026 & -0.017 & +0.018 & +0.074 \\
\hline
\end{tabular}

6. Generalized weighted sums. Let (a) again denote a set of distinct positive numbers $a_{1}, a_{2}, \cdots, a_{n}$ and $(\xi)$ a set of arbitrary positive numbers $\xi_{1}, \xi_{2}, \cdots, \xi_{n}, n \geqq 2$. Consider the function $S_{t}(a, \xi)$ 
$=\left[\sum_{\nu=1}^{n} \xi_{\nu} a_{\nu}^{2}\right]^{1 / t}$ for $t \neq 0, \pm \infty$;

$$
\begin{aligned}
S_{0}(a, \xi) & =\prod_{\nu=1}^{n} a_{\nu}^{\xi_{\nu}} \quad \text { if } \sum_{\nu=1}^{n} \xi_{\nu}=1 ; \\
S_{0}(a, \xi) & =0 \text { if } \sum_{\nu=1}^{n} \xi_{\nu} \neq 1 ; \\
S_{-\infty}(a, \xi) & =\min _{\nu=1,2, \ldots, n} a_{\nu}
\end{aligned}
$$

and

$$
S_{+\infty}(a, \xi)=\max _{\nu=1,2, \ldots, n} a_{\nu}
$$

For fixed sets $(a)$ and $(\xi)$, the function $S_{t}(a, \xi)$ is continuous for $-\infty \leqq t<0$ and for $0<t \leqq+\infty$. If $\sum_{\nu=1}^{n} \xi_{\nu} \leqq 1$, then $S_{t}(a, \xi)$ is continuous from the right at $t=0$; and if $\sum_{\nu=1}^{n} \xi_{\nu} \geqq 1$, then $S_{t}(a, \xi)$ is continuous from the left at $t=0$. However, if we define $f, \eta_{\nu}$, and $S_{k}$ as in formulae (1), (2), and (3), then all of the results of $\$ 3$ regarding $f^{\prime \prime \prime}(t)$ are valid without modification. Formula (7) will hold as long as $t_{1}$ and $t_{2}$ are both positive or both negative.

THEOREM 4. (a) There is a point $p_{1}$ such that $\log S_{t}(a, \xi)$ is either $a$ concave function of $t$ for all $t<p_{1}$ or a convex function for all $t<p_{1}$.

(b) There is a point $p_{2}$ such that $\log S_{t}(a, \xi)$ is convex for all $t>p_{2}$, or concave for all $t>p_{2}$.

P'roof of Theorem 4a. Assume that for all $p, \log S_{t}(a, \xi)$ is not convex in the range $-\infty<t<p$. Let $p_{1}<0$ be such that $t<p_{1}$ implies that $f^{\prime \prime \prime}(t)>0$. Consider any number $t_{0}<p_{1}$. Then, by assumption, $d^{2} \log S_{t}(a, \xi) / d t^{2}<0$ has a solution for some $t^{\prime}<t_{0}$. Formula (7) implies that this second derivative is negative for all $t$ satisfying $t^{\prime}<t \leqq p_{1}$, or that $\log S_{t}(a, \xi)$ is concave for all $t \leqq p_{1}$.

The proof of Theorem $4 \mathrm{~b}$ is similar.

To show the applicability of Theorem 4 consider a function $S_{t}(a, \xi)$ where $\sum_{\nu=1}^{n} \xi_{\nu}<1$. For all such sets $(\xi)$ and for every set $(a)$, the function $S_{t}(a, \xi)$ has the following properties:

(a) $t_{1}<t_{2}$ and $t_{1} t_{2}>0$ imply that $S_{t_{1}}(a, \xi)<S_{t_{2}}(a, \xi)$;

(b) $\min _{\nu=1,2, \cdots, n} a_{\nu}<S_{t}(a, \xi)$ for $t<0$;

(c) $S_{t}(a, \xi)<\max _{\nu=1,2, \cdots, n} a_{\nu}$ for $t>0$.

Hence there is no point $p$ such that $t<p$ implies that $\log S_{t}(a, \xi)$ is concave nor a point $p$ such that $t>p$ implies that $\log S_{t}(a, \xi)$ is convex. We conclude from Theorem 4 that if $\sum_{\nu=1}^{n} \xi_{\nu}<1, \log S_{t}(a, \xi)$ is convex in a neighborhood of $-\infty$ and concave in a neighborhood of $+\infty$. 
If $\xi_{\nu} \geqq 1$ for each $\nu$, then by reasoning analogous to that of the preceding example, it may be shown, for any set $(a)$, that there is no point $p$ such that $t<p$ implies that $\log S_{t}(a, \xi)$ is convex nor a point $p$ such that $t>p$ implies that $\log S_{t}(a, \xi)$ is concave. Hence Theorem 4 applies to all such functions $\log S_{t}(a, \xi)$. However, for this case the conclusion of the general theorem is weaker than the known result that $\log S_{t}(a, \xi)$ is convex for all positive $t$ and concave for all negative $t .^{2}$

University of California at Los Angeles

${ }^{2}$ See Beckenbach, An inequality of Jensen, Amer. Math. Monthly vol. 53 (1946) pp. 501-505.

\section{HOMOMORPHISMS ON BANACH SPACES}

\section{E. MUNROE}

1. Introduction. Let $E$ be a Banach space and $E^{*}$ its conjugate space. Let $G$ be a closed linear subspace of $E$, and let $\Gamma=\left\{f \mid f \in E^{*}\right.$, $f(x)=0$ for $x \in G\}$. Krein and Smulian have shown $\left[4 \text {, Theorem } 12^{\prime}\right]^{1}$ that $G^{*}=E^{*} / \Gamma$ in the sense that the two spaces are algebraically isomorphic and that the usual definitions of norm in the two are equivalent. Noting the algebraic isomorphism, let us look at the topological aspects of this equivalence in a slightly different light. $G^{*}$ being a factor space of $E^{*}$, there is defined a natural homomorphism [5, p. 64] $T\left(E^{*}\right)=G^{*}$. Since they are using the induced topology $[5, \mathrm{p}$. 58] in $E^{*} / \Gamma$, Krein and Smulian prove what is equivalent to the theorem that the transformation $T$ is continuous and open (see [5, Theorem 12]). Stated in this way, incidentally, the result follows immediately from the Hahn-Banach theorem by means of the usual neighborhood argument for continuity and openness.

However, the homomorphism $T\left(E^{*}\right)=G^{*}$ suggests other topological questions the answers to which are not quite so obvious. Specifically, what are the topological properties of $T$ when $E^{*}$ and $G^{*}$ are given topologies other than their norm topologies?

The conjugate to a Banach space may be topologized in any one of several well known ways. The most common such topologies are the norm, weak, weak*, bounded weak and bounded weak*. We shall

Presented to the Society, October 25, 1947; received by the editors September 10, 1947.

${ }^{1}$ Numbers in brackets refer to the bibliography at the end of the paper. 